\title{
METRICAL THEOREMS ON FRACTIONAL PARTS OF SEQUENCES
}

\author{
BY \\ WOLFGANG M. SCHMIDT(1)
}

1. Introduction. Let $C$ be the additive group of real numbers modulo 1 , and let $x \rightarrow\{x\}$ be the natural mapping from the reals onto $C$. It is clear what we shall mean by an interval $I$ in $C$ and by the length $l(I)$ of $I$. Denote the distance of the real number $\alpha$ to the closest integer by $\|\alpha\|$. The image in $C$ of the set of reals $\xi$ satisfying $\|\xi-\theta\| \leqq \varepsilon$ with given $\theta$ and $0<\varepsilon<1 / 2$ is an example of an interval of $C$ of length $2 \varepsilon$.

THEOREM 1. Let $n \geqq 1$ and let $P_{1}(q), \cdots, P_{n}(q)$ be nonconstant polynomials with integral coefficients. For each of the integers $j=1, \cdots, n$ let $I_{j 1} \supseteq I_{j 2} \supseteq \cdots$ be a sequence of nested intervals in C. Put $\psi(q)=l\left(I_{1 q}\right) \cdots l\left(I_{n q}\right)$ and

$$
\Psi(h)=\sum_{q=1}^{h} \psi(q) .
$$

Put $N\left(h ; \alpha_{1}, \cdots, \alpha_{n}\right)$ for the number of integers $q, 1 \leqq q \leqq h$, with

$$
\left\{\alpha_{j} P_{j}(q)\right\} \in I_{j q}
$$$$
(j=1, \cdots, n)
$$

Let $\varepsilon>0$. Then

$$
N\left(h ; \alpha_{1}, \cdots, \alpha_{n}\right)=\Psi(h)+O\left(\Psi(h)^{1 / 2+\varepsilon}\right)
$$

for almost every $n$-tuple of real numbers $\alpha_{1}, \cdots, \alpha_{n}$.

The theorem implies, for example, that the number of solutions of

$$
|\alpha q-p-\theta| \leqq q^{-1}
$$

in integers $p$ and $q, 1 \leqq q \leqq h$, is asymptotically equal to $2 \log h$ for every $\alpha \notin \sigma(\theta)$ where $\sigma(\theta)$ is a set of measure zero. To see this we only have to put $n=1, P(q)=q$ and to define intervals $I_{q}$ as the images of the sets $\|\xi-\theta\| \leqq q^{-1}$.

On the other hand, let $P(q)=a_{0} q^{d}+\cdots+a_{d}$ be a polynomial of degree $d>0$ with integral coefficients, let $\mu$ be real, and let $M(h ; \alpha)$ be the number of solutions in integers $p, q, 1 \leqq q \leqq h$, of

$$
|\alpha-p / P(q)| \leqq q^{-\mu} .
$$

Received by the editors August 29, 1962 and, in revised form, December 11, 1962.

(1) This paper was written with partial support of the National Science Foundation Grant No. NSF-G19894. 
Then $M(h ; \alpha)$ is bounded for almost every $\alpha$ if $\mu>d+1 ; M(h ; \alpha) \sim 2\left|a_{0}\right| \log h$ if $\mu=d+1$; and $M(h ; \alpha) \sim 2\left|a_{0}\right| h^{d+1-\mu}(d+1-\mu)^{-1}$ for almost every $\alpha$ if $\mu<d+1$.

To see this, we remark that for $\mu>d$ and large $q,(1.4)$ is equivalent to $\|\alpha P(q)\| \leqq|P(q)| q^{-\mu}$. Thus our interval $I_{q}$ has length $\psi(q)=2|P(q)| q^{-\mu}$ $=\left|2 a_{0} q^{d-\mu}+2 a_{1} q^{d-\mu-1}+\cdots\right|$, and the theorem gives the result. For $\mu=d,(1.4)$ becomes $|\alpha P(q)-p| \leqq\left|a_{0}+a_{1} q^{-1}+\cdots\right|$, and $M(h ; \alpha)$ becomes $2\left|a_{0}\right| h$ plus (or minus) the number of solutions of $\|\alpha P(q)\| \leqq\left|a_{1} q^{-1}+\cdots\right|$ for $1 \leqq q \leqq h$, whence $M(h ; \alpha) \sim 2\left|a_{0}\right| h$ almost everywhere. Finally for $\mu<d$ our formula for $M(h ; \alpha)$ is in fact true for every $\alpha$. The reader should have no difficulty in proving this elementary result.

There can be at most countably many $\alpha_{j}$ 's such that $\left\{\alpha_{j} P_{j}(q)\right\}$ is an endpoint of $I_{j q}$ for some $q$, and hence we may assume $I_{j q}$ to be $\operatorname{closed}(j=1, \cdots, n ; q=1,2, \cdots)$. The intersections $J_{j}=\bigcap_{q} I_{j q}(j=1, \cdots, n)$ are then nonempty. The case where $0 \in J_{j}$ for each $j$ is usually called the homogeneous case, the general case the inhomogeneous case.

Our theorem implies in particular that $N\left(h ; \alpha_{1}, \cdots, \alpha_{n}\right)$ remains bounded almost everywhere if $\Psi(h)$ is bounded, while it will tend to infinity almost everywhere if $\Psi(h)$ tends to infinity. This had keen proved by Khintchine [9] in the homogeneous case under the assumption that $P_{j}(q)=q(j=1, \cdots, n)$ and that $q \psi(q)$ is decreasing. Szüsz [13] generalized Khintchine's result to the inhomogeneous case. Szüsz' method involves continued fractions and therefore applies only to the case $n=1$. Before Szüsz, Cassels [2] had shown that Khintchine's conclusion is true for "almost every inhomogeneous case," that is, if $\left(I_{1 q}, \cdots, I_{n q}\right)$ is replaced by its translation by a vector $\left(\theta_{1}, \cdots, \theta_{n}\right)$ of reals $\bmod 1(q=1,2, \cdots)$, then the conclusion is true for almost every $\theta_{1}, \cdots, \theta_{n}$. Thus Cassel's result was "doubly metrical."

Erdös [5] proved for the homogeneous case with $n=1, P(q)=q$, that $N(h ; \alpha) \sim \Psi(h)$ almost everywhere, and the author $[12]\left({ }^{2}\right)$ proved (1.3) in this case. Our generalization from the homogeneous to the inhomogeneous case is not trivial. We shall choose $\theta_{j} \in J_{j}(j=1, \cdots, n)$ and use rational approximations to $\theta_{j}$. The generalization from linear to general polynomials also causes some difficulty.

Le Veque [10] proved a general theorem where polynomials $P(q)$ are replaced by general sequences $a(q)$ which have to satisfy a certain condition. However, this condition is not satisfied for $a(q)=q$, and it is difficult to decide whether it is satisfied for nonlinear polynomials.

It would be possible to replace (1.2) by $\left(\left\{\alpha_{1} P_{1}(q)\right\}, \cdots,\left\{\alpha_{n} P_{n}(q)\right\}\right) \in H_{q}$, thus replacing products of intervals $I_{1 q} \times \cdots \times I_{n q}$ by somewhat more general sets $H_{q}$ of $C \times \cdots \times C$.

(2) We use this opportunity to mention two errors in [12]: In Theorem 1 of [12] one has to assume that the functions $\psi_{j}(q)$ are bounded. Everywhere in $\S 6$ except in $\beta(Q, \theta), \theta$ should be replaced by $\Theta=\left(\theta_{1}, \ldots, \theta_{u}\right)$. 
In $\$ 10$ we shall point out how one could prove a more general theorem where the expressions $\alpha_{j} P_{j}(q)$ are replaced by linear forms $\alpha_{j 1} P_{j 1}\left(q_{1}\right)+\cdots+\alpha_{j m} P_{j m}\left(q_{m}\right)$. A special case of such a result is contained in Theorem 2 of [12].

THEOREM 2. Let a sequence of positive integers $a_{i}(1)<a_{i}(2)<\cdots$ be given for $i=1, \cdots, n$. Let $\theta$ be arbitrary but fixed, and put

$$
\sum\left(h ; \alpha_{1}, \cdots, \alpha_{n}\right)=\sum_{q_{1}=1}^{h} \ldots \sum_{q_{n}=1}^{h}\left(q_{1} \cdots q_{n}\left\|\sum_{i=1}^{n} \alpha_{i} a_{i}\left(q_{i}\right)+\theta\right\|\right)^{-1} .
$$

Then one has for $\varepsilon>0$ and almost every $\alpha_{1}, \cdots, \alpha_{n}$

$$
(\log h)^{n+1} \ll \Sigma\left(h ; \alpha_{1}, \cdots, \alpha_{n}\right) \ll(\log h)^{n+1+\varepsilon} .
$$

Using Theorem 2, together with an $n$-dimensional generalization of a result of Erdös and Turan [7, Theorem 3], we shall easily deduce

THEOREM 3. Assume the hypotheses of Theorem 1 to be satisfied, and assume we deal with the special case $P_{j}(q)=q(j=1, \cdots, n)$ and $I_{j 1}=I_{j 2}$ $=\cdots(j=1, \cdots, n)$. Write $\psi$ for $\psi(1)=\psi(2)=\cdots$, and let $\varepsilon>0$. Then

for almost every $\alpha_{1}, \cdots, \alpha_{n}$.

$$
N\left(h ; \alpha_{1}, \cdots, \alpha_{n}\right)=h \psi+O(\log h)^{n+1+\varepsilon}
$$

Khintchine $[8, \S 3]$, proved the surprisingly small error-term $O(\log h)^{1+\varepsilon}$ for $n=1$, and hence our result is not best possible. However, Khintchine's method involves continued fractions and cannot easily be generalized to $n>1$. It seems that Theorem 1 cannot much be improved for nonlinear polynomials. Behnke [1, Theorem XXV] showed for $n=1, P(q)=q^{2}$ and $I_{1}=I_{2}=\cdots=I$, say, that the relation $D_{\alpha}(h)={ }^{\text {def }} \sup _{I}\left|N_{I}(h ; \alpha)-h l(I)\right| \ll \sqrt{ } h$ is wrong for every $\alpha$.

2. Notation and simplification. Throughout, $[\alpha]$ will the integral part of the real number $\alpha$. $U$ will denote the unit interval $0 \leqq \xi<1$.

We shall prove the case $n=1$ of Theorem 1 in $\S \S 2-8$. In $\S 9$ we shall point out the necessary changes for $n>1$.

The set of $\alpha$ 's in $U$ where $\{\alpha P(q)\} \in I_{q}$ has measure $\psi(q)$. Assume now that $\Psi(h)$ is bounded. Given $\varepsilon>0$ there is a $q_{0}$ such that $\sum_{q>q_{0}} \psi(q)<\varepsilon$, and the set of $\alpha$ 's in $U$ such that $\{\alpha P(q)\} \in I_{q}$ for some $q>q_{0}$ has measure $<\varepsilon$. Hence $N(h ; \alpha)$ is bounded for almost every $\alpha$.

From now on, we shall assume that $\Psi(h)$ tends to infinity.

Let $\theta \in J=\bigcap I_{q}$. Then each $I_{q}$ is union of $\theta$ and of two intervals $I_{q}^{l}$ and $I_{q}^{r}$, where $I_{q}^{l}$ is of the type $0<\{\theta-\xi\} \leqq \psi^{l}(q)$, where $I_{q}^{r}$ is of the type $0<\{\xi-\theta\} \leqq \psi^{r}(q)$, and where $\psi^{l}(q)+\psi^{r}(q)=\psi(q)$. ( $I_{q}^{l}$ or $I_{q}^{r}$ may be empty.) Now $\Psi^{l}(h), \Psi^{r}(h)$, $N^{l}(h ; \alpha), N^{r}(h ; \alpha)$ can be defined in the obvious way. One has $\Psi(h)=\Psi^{l}(h)+\Psi^{r}(h)$ and $N(h ; \alpha)=N^{l}(h ; \alpha)+N^{r}(h ; \alpha)$ for almost every $\alpha$. Hence it will suffice to prove the theorem for the case of intervals of type $I^{l}$ and the case of intervals of type $I^{r}$. 
Since the mapping $\xi \rightarrow-\xi, \theta \rightarrow-\theta$ transforms intervals of type $I^{l}$ into intervals of type $\boldsymbol{I}^{r}$, we may restrict ourselves to intervals of type $\boldsymbol{I}^{r}$.

From now on, $I_{q}$ will denote the interval

$$
0<\{\xi-\theta\} \leqq \psi(q) .
$$

Replacing $P(q)$ by $-P(q)$ and $\alpha$ by $-\alpha$ if necessary, we may assume that $P(q)>0, P^{\prime}(q)>0$ for $q>q_{0}$. Making a translation by $q_{0}$ we may even assume $P(q)>0, P^{\prime}(q)>0$ for $q>0$.

The introduction of a parameter $k$ is essential for our proof. Put $\phi(k, x)$ for the number of integers $y$ between 1 and $x, 1 \leqq y \leqq x$, such that g.c.d. $(x, y) \leqq k$. $\phi(1, x)$ is the well-known Euler $\phi$-function.

Given $q \geqq 1$ there are pairs of relatively prime integers $a, b$ such that

$$
1 \leqq a \leqq q^{1 / 2} \text { and }|\theta-b / a|<a^{-1} q^{-1 / 2} \text {. }
$$

This follows from Dirichlet's principle. For every integer $q \geqq 1$ we pick integers $a=a(q), b=b(q)$ with these properties. We define $S(k, q)$ as the set of integers $p$ where

$$
\text { g.c.d. }(p a(q)+b(q), P(q)) \leqq k .
$$

The sets $S(k, q)$ have two important properties:

(1) If $p \in S(k, q)$ and $p \equiv p^{\prime}(\bmod P(q))$, then $p^{\prime} \in S(k, q)$.

(2) The number $\phi^{*}(k, q)$ of integers of $S(k, q)$ in $1 \leqq x \leqq P(q)$ satisfies $\phi^{*}(k, q) \geqq \phi(k, P(q))$.

To prove (2), put $P(q)=q_{1} q_{2}$ where every prime factor of $q_{1}$ divides $a$ and where $q_{2}$ and $a$ are relatively prime. Now g.c.d. $(a, b)=1$ yields g.c.d. $(p a+b, P(q))=$ g.c.d. $\left(p a+b, q_{2}\right)$ and $\phi^{*}(k, q)=q_{1} \phi\left(k, q_{2}\right) \geqq \phi(k, P(q))$.

We now put

$$
\begin{aligned}
\beta(q, \alpha) & =\left\{\begin{array}{l}
1 \text { if } \alpha \in U \text { and }\{\alpha\} \in I_{q}, \\
0 \text { otherwise, }
\end{array}\right. \\
\gamma(q, \alpha) & =\sum_{p} \beta(q, \alpha P(q)-p), \\
\gamma(k, q, \alpha) & =\sum_{p \in S(k, q)} \beta(q, \alpha P(q)-p), \\
\Gamma(q) & =\int_{0}^{1} \gamma(q, \alpha) d \alpha \\
\Gamma(k, q) & =\int_{0}^{1} \gamma(k, q, \alpha) d \alpha, \\
\Gamma(k, q, r) & =\int_{0}^{1} \gamma(k, q, \alpha) \gamma(k, r, \alpha) d \alpha, \\
A(k, q, r) & =\Gamma(k, q, r)-\psi(q) \psi(r),
\end{aligned}
$$


and

$$
\Psi(u, v)=\sum_{q=u+1}^{v} \psi(q) .
$$

It is easy to see that $N(h ; \alpha)=\sum_{q=1}^{h} \gamma(q, \alpha)$, and we define

$$
N(k ; u, v ; \alpha)=\sum_{q=u+1}^{v} \gamma(k, q, \alpha) .
$$

One has

$$
\Gamma(q)=\sum_{p} \int_{0}^{1} \beta(q, P(q) \alpha-p) d \alpha=P(q) \int_{-\infty}^{\infty} \beta(q, P(q) \alpha) d \alpha=\psi(q),
$$

and similarly

$$
\Gamma(k, q)=\psi(q) \phi^{*}(k, q) P(q)^{-1} .
$$

Summing over $q$ we find

$$
\int_{0}^{1} N(h ; \alpha) d \alpha=\Psi(h)
$$

and

$$
\int_{0}^{1} N(k ; u, v ; \alpha) d \alpha=\sum_{q=u+1}^{v} \psi(q) \phi^{*}(k, q) P(q)^{-1}
$$

\section{Deduction of Theorem 1 from two propositions.}

Proposition 1. Let $\delta>0$. Then

$$
\sum_{q=1}^{h}(P(q)-\phi(k, P(q))) P(q)^{-1} \ll h k^{\delta-1}+h^{\delta} k^{\delta}
$$

Proposition 2. For every $\delta>0$

$$
\sum_{q=1}^{h} \sum_{r=1}^{h} A(k, q, r) \ll \Psi^{1+\delta}(h)+\Psi(h) k^{\delta} .
$$

REMARK. Here and later, the estimate $\ll$ holds simultaneously in $h$ and $k$. That is, the constant implied by $\ll$ depends only on $\delta$.

We are going to show that Theorem 1 is a consequence of these two propositions. The propositions will be proved later.

LEMMA 1. Let $\omega_{1}(q), \omega_{2}(q), \omega_{3}(q)$ be positive bounded functions of positive integers $q$, and put

$$
\Omega_{i}(h)=\sum_{q=1}^{h} \omega_{i}(q) \quad(i=1,2,3)
$$


Assume that $\omega_{1}$ and $\omega_{3}$ are decreasing, and that $\Omega_{2}(r) \leqq \Omega_{3}(r)$ for every $r$. Then

$$
\sum_{q=1}^{h} \omega_{1}(q) \omega_{2}(q) \ll \Omega_{3}\left(\left[\Omega_{1}(h)\right]\right) .
$$

Proof. If $\Omega_{1}$ is bounded, then so is the sum in (3.3). Hence we assume $\Omega_{1}$ to be unbounded. Since $\omega_{1}$ is decreasing, and since $\Omega_{2} \leqq \Omega_{3}$, one finds by partial summation that $\sum_{q=1}^{h} \omega_{1}(q) \omega_{2}(q) \leqq \sum_{q=1}^{h} \omega_{1}(q) \omega_{3}(q)$.

To estimate the latter sum we may assume $\omega_{1}(q) \leqq 1$. Put $m_{0}=0$ and for integral $a>0$ put $m_{a}$ for the largest $m$ with $\Omega_{1}(m) \leqq a$. Then $m_{a} \geqq a$ and $\omega_{1}\left(m_{a}+1\right)+\cdots+\omega_{1}\left(m_{a+1}\right) \leqq 2$. Putting $b=\left[\Omega_{1}(h)\right]$ we obtain

$$
\begin{aligned}
\sum_{q=1}^{h} \omega_{1}(q) \omega_{3}(q) & \leqq \sum_{a=0}^{b}\left(\omega_{1}\left(m_{a}+1\right) \omega_{3}\left(m_{a}+1\right)+\cdots+\omega_{1}\left(m_{a+1}\right) \omega_{3}\left(m_{a+1}\right)\right) \\
& \leqq 2 \sum_{a=0}^{b} \omega_{3}\left(m_{a}+1\right) \leqq 2 \sum_{a=1}^{b+1} \omega_{3}(a)=2 \Omega_{3}\left(\left[\Omega_{1}(h)\right]+1\right) .
\end{aligned}
$$

Denote by $J_{r}$ the set of intervals $(u, v], 0 \leqq u=t \cdot 2^{s}<v=(t+1) 2^{s} \leqq 2^{r}$ where $r, s, t$ are non-negative integers. Every interval $(0, w]$ where $w$ is integral and $w \leqq 2^{r}$ is union of not more than $\max (1, r)$ intervals of $J_{r}$. Given an integer $u>0$ put $n_{u}$ for some integer satisfying $\left[\Psi\left(n_{u}\right)\right]=u$, and put $n_{0}=0$. Since $\psi(q) \leqq 1$ and since $\Psi(h)$ tends to infinity, such an $n_{u}$ will always exist. Put $h_{r}=n_{2}$.

For the remainder of this section, $k$ and $r$ will be connected by

$$
k=2^{r} \text {. }
$$

LEMMA 2. Let $\delta>0$. Then

$$
0 \leqq \int_{0}^{1}\left(N\left(h_{r} ; \alpha\right)-N\left(k ; 0, h_{r} ; \alpha\right)\right) d \alpha \ll 2^{r \delta}
$$

and

$$
\sum_{(u, v] \in J_{r}} \int_{0}^{1}\left(N\left(k ; n_{u}, n_{v} ; \alpha\right)-\Psi\left(n_{u}, n_{v}\right)\right)^{2} d \alpha \ll 2^{r+r \delta} .
$$

Proof. Formulae (2.5) and (2.6) yield

$$
\begin{aligned}
S_{r} & \equiv \int_{0}^{1}\left(N\left(h_{r} ; \alpha\right)-N\left(k ; 0, h_{r} ; \alpha\right)\right) d \alpha=\sum_{q=1}^{h_{r}} \psi(q)\left(P(q)-\phi^{*}(k, q)\right) P(q)^{-1} \\
& \leqq \sum_{q=1}^{h_{r}} \psi(q)(P(q)-\phi(k, P(q))) P(q)^{-1} .
\end{aligned}
$$

We now put

$$
\omega_{1}(q)=\psi(q), \omega_{2}(q)=(P(q)-\phi(k, P(q))) P(q)^{-1}, \omega_{3}(q)=c\left(k^{\delta-1}+q^{\delta-1} k^{\delta}\right) .
$$

Proposition 1 shows that Lemma 1 is applicable if $c>0$ is chosen large enough. Under our conditions we actually obtain the bound $\left.2 \Omega_{3}\left(\left[\Omega_{1}(h)\right)\right]+1\right)$. Hence 


$$
S_{r} \ll \Psi\left(h_{r}\right) k^{\delta-1}+\Psi\left(h_{r}\right)^{\delta} k^{\delta} \ll 2^{r+r(\delta-1)}+2^{2 r \delta} \ll 2^{2 r \delta} .
$$

This is true for every $\delta>0$, and hence (3.5) is proved.

$$
N(k ; u, v ; \alpha)-\Psi(u, v)=\sum_{q=u+1}^{v}(\gamma(k, q, \alpha)-\psi(q)) .
$$

Hence by (2.3), (2.4) and the estimate just derived,

$$
\begin{aligned}
\int_{0}^{1} & (N(k ; u, v ; \alpha)-\Psi(u, v))^{2} d \alpha \\
& =\sum_{q=u+1}^{v} \sum_{q^{\prime}=u+1}^{v}\left(\Gamma\left(k, q, q^{\prime}\right)-\Gamma(k, q) \psi\left(q^{\prime}\right)-\Gamma\left(k, q^{\prime}\right) \psi(q)+\psi(q) \psi\left(q^{\prime}\right)\right) \\
& =\sum_{q=u+1}^{v} \sum_{q^{\prime}=u+1}^{v} A\left(k, q, q^{\prime}\right)+2 \sum_{q=u+1}^{v} \sum_{q^{\prime}=u+1}^{v} \psi(q) \psi\left(q^{\prime}\right)\left(P(q)-\phi^{*}(k, q)\right) P(q)^{-1} \\
& \ll \sum_{q=u+1}^{v} \sum_{q^{\prime}=u+1}^{v} A\left(k, q, q^{\prime}\right)+\sum_{q^{\prime}=u+1}^{v} \psi\left(q^{\prime}\right) 2^{r \delta} .
\end{aligned}
$$

We first consider the part of the sum (3.6) where $(u, v]$ are intervals of $J_{r}$ with fixed $s$ (see the definition of $J_{r}$ ). These intervals cover $\left(0,2^{r}\right]$ exactly once, and hence the corresponding intervals $\left(n_{u}, n_{v}\right]$ cover $\left(0, h_{r}\right]$ exactly once. Our part of the sum (3.6) has the upper bound

$$
\sum_{q=1}^{h_{r}} \sum_{q^{\prime}=1}^{h_{r}} A\left(k, q, q^{\prime}\right)+2^{r \delta} \sum_{q=1}^{h_{r}} \psi(q) \ll \Psi\left(h_{r}\right)^{1+\delta}+\Psi\left(h_{r}\right) k^{\delta}+2^{r \delta} \Psi\left(h_{r}\right) \ll 2^{r+r \delta} .
$$

Summing over $s$ from 0 to $r$ we find the bound $\ll r 2^{r+r \delta}$ for the sum (3.6). Since $\delta>0$ is arbitrary, Lemma 2 is proved.

LEMMA 3. Let $\delta$ be positive and fixed. Then there is a sequence of subsets $\sigma_{1}, \sigma_{2}, \cdots$ of $U$ with measures

such that

$$
\mu_{r}=\int_{\sigma r} d \alpha \ll r^{-2}
$$

$$
N\left(n_{w} ; \alpha\right)=\Psi\left(n_{w}\right)+O\left(r^{2} 2^{r / 2+r \delta}\right)
$$

for every $w \leqq 2^{r}$ and every $\alpha \in U$ which is not in $\sigma_{r}$.

Proof. We define $\sigma_{r}$ to be the subset of $U$ where not both of the following inequalities hold:

$$
\begin{gathered}
0 \leqq N\left(h_{r} ; \alpha\right)-N\left(k ; 0, h_{r} ; \alpha\right) \leqq r^{2} 2^{r / 2} \\
\sum_{(u, v] \in J_{r}}\left(N\left(k ; n_{u}, n_{v} ; \alpha\right)-\Psi\left(n_{u}, n_{v}\right)\right)^{2} \leqq r^{2} 2^{r+r \delta}
\end{gathered}
$$


Lemma 2 implies $\mu_{r} \ll r^{-2}$. Every interval $(0, w], w \leqq 2^{r}$, is union of at most $\max (1, r)$ intervals of $J_{r}$, hence $\left(0, n_{w}\right]$ is union at most $\max (1, r)$ intervals $\left(n_{u}, n_{v}\right]$ where $(u, v] \in J_{r}$. Thus $N\left(k ; 0, n_{w} ; \alpha\right)-\Psi\left(n_{w}\right)=\Sigma\left(N\left(k ; n_{u}, n_{v} ; \alpha\right)-\Psi\left(n_{u}, n_{v}\right)\right)$, where the sum is over at most $r+1$ pairs $(u, v] \in J_{r}$. This relation together with (3.8) and Cauchy's inequality gives for $\alpha \in U, \alpha \notin \sigma_{r}$

$$
\left(N\left(k ; 0, n_{w} ; \alpha\right)-\Psi\left(n_{w}\right)\right)^{2} \leqq r^{2}(r+1) 2^{r+r \delta} .
$$

Lemma 3 is a consequence of (3.7)=and (3.9).

Proof of Theorem 1. Since $\sum r^{-2}$ is convergent, there exists for almost every $\alpha \in U$ an $r_{0}=r_{0}(\alpha)$ such that $\alpha \notin \sigma_{r}$ for $r \geqq r_{0}$. Assume $\alpha$ has such an $r_{0}$, and assume $w>2^{r 0}$. Choose $r$ such that $2^{r-1} \leqq w<2^{r}$. Then $r>r_{0}, \alpha \notin \sigma_{r}$, and Lemma 3 implies

$$
\begin{aligned}
N\left(n_{w} ; \alpha\right) & =\Psi\left(n_{w}\right)+O\left(r^{2} 2^{r / 2+r \delta}\right) \\
& =\Psi\left(n_{w}\right)+O\left(w^{1 / 2+\delta} \log ^{2} w\right) \\
& =\Psi\left(n_{w}\right)+O\left(\Psi^{1 / 2+\delta}\left(n_{w}\right) \log ^{2} \Psi\left(n_{w}\right)\right) .
\end{aligned}
$$

Since $\Psi\left(n_{w+1}\right)=\Psi\left(n_{w}\right)+O(1),(3.10)$ is true for arbitrary integers $h$ and not only the $n_{w}$ 's. And since $\delta>0$ was arbitrary, we find

$$
N(h ; \alpha)=\Psi(h)+O\left(\Psi(h)^{1 / 2+\varepsilon}\right)
$$

for almost every $\alpha \in U$. Hence (1.3) is true for almost every $\alpha$.

4. The number of solutions of $P(x) \equiv 0(\bmod d)$. Put $D(q)$ for the number of positive divisors of $q$. As is well known,

$$
D(q) \ll q^{\delta}
$$

for every $\delta>0$. Put $z(d)=z_{P}(d)$ for the number of solutions of $P(x) \equiv 0(\bmod d)$. Here, as always, $P(x)$ is a nonconstant polynomial with integral coefficients. Define the discriminant $\Delta$ of $P(x)$ in the usual way if $P(x)$ is nonlinear, and put $\Delta=\alpha_{0}$ if $P(x)=a_{0} x+a_{1}$.

LeMma 4. Let $P(x)$ be a polynomial of degree $f$ and with discriminant $\Delta \neq 0$. Then $z_{P}\left(p^{k}\right) \leqq f \Delta^{2}$ for every prime-power $p^{k}$.

Proof. For linear $P(x)$ it is well known that $z(m) \leqq$ g.c.d. $(m, \Delta) \leqq \Delta \leqq f \Delta^{2}$. The case where $P(x)$ is nonlinear and primitive, that is, where the coefficients of $P(x)$ are relatively prime, is Theorem 54 of [11]. A proof can be found there. In the general nonlinear case one has $P(x)=c Q(x)$ with primitive $Q(x)$, whence $z_{P}\left(p^{k}\right) \leqq c z_{Q}\left(p^{k}\right) \leqq c f \Delta^{2}{ }_{Q} \leqq f \Delta^{2}{ }_{P}$.

Corollary. Let $P(x)$ be a polynomial with no multiple factors. Let $\delta>0$. Then 


$$
z_{P}(d) \ll d^{\delta} .
$$

Proof. The set $\tau$ of prime-powers $p^{k}$ such that $p^{k \delta} \bar{\Sigma} f \Delta^{2}$ is finete. For every $d$,

$$
z(d) d^{-\delta} \leqq \prod_{p^{k} \in \tau} z\left(p^{k}\right) p^{-k \delta} \ll 1 .
$$

Given an integer $g>0$ we define a function ${ }^{g}(d)$ of positive integers $d$ as follows: ${ }^{g}(d)$ is multiplicative, and ${ }^{g}\left(p^{g x+y}\right)=p^{x+1}$ if $p$ is a prime and $1 \leqq y \leqq g$. Our function has the property that $d \mid m^{g}$ implies $^{g}(d) \mid m$.

LEMma 5. Let $P(x)$ be a nonconstant polynomial, $g$ a positive integer and $s>1$. Then the two sums

$$
\sum_{d=1}^{\infty} z_{P}(d) d^{-s}
$$

and

$$
\sum_{d=1}^{\infty}\left({ }^{g}(d)\right)^{-s}
$$

are convergent.

Proof. There is an integer $m$ and a polynomial $Q(x)$ without multiple factors such that $P(x) \mid Q(x)^{m}$. Now $P(x) \equiv 0(\bmod d)$ implies $Q(x) \equiv 0\left(^{m}(d)\right)$, and hence $\left.z_{P}(d) d^{-1} \leqq z_{Q} Q^{m}(d)\right)\left(^{m}(d)\right)^{-1}$. Thus one has for $d=p^{m x+y}$ where $p$ is prime and $0<y \leqq m$,

$$
\begin{aligned}
z_{P}(d) d^{-s} & \leqq z_{Q}\left(^{m}(d)\right)\left(^{m}(d)\right)^{-1} d^{1-s}=z_{Q}\left(p^{x+1}\right) p^{(1-s)(m x+y)-x-1} \\
& \leqq f \Delta_{Q}^{2} p^{-x(m(s-1)+1)-y(s-1)-1} \leqq f \Delta_{Q}^{2} p^{-s x-s}
\end{aligned}
$$

This implies

$$
\sum_{e=1}^{\infty} z_{P}\left(p^{e}\right) p^{-e s} \leqq m f \Delta_{Q}^{2} p_{s}^{-s} \sum_{x=0}^{\infty} p^{-x s} \leqq c_{s} p^{-s} .
$$

Since the product $\prod_{p}\left(1+c p^{-s}\right)$ over all primes $p$ is convergent, the convergence of (4.3) follows.

The convergence of (4.4) is proved similarly.

5. Proof of Proposition 1. The Euler $\phi$-function $\phi(x)=\phi(1, x)$ can be expressed $\phi(x)=x \Sigma_{y \mid x} \mu(y) y^{-1}$, where $\mu(y)$ is the Moebius function. Now hence

$$
\phi(k, P(q))=\sum_{x \leqq k ; x \mid P(q)} \phi\left(P(q) x^{-1}\right)=\sum_{x \leqq k ; x \mid P(q)} P(q) x^{-1} \sum_{y \mid P(q) x^{-1}} \mu(y) y^{-1} ;
$$

$$
\begin{aligned}
T_{k, h} & \equiv \sum_{q=1}^{h} \phi(k, P(q)) P(q)^{-1}=\sum_{q=1}^{h} \sum_{x \leqq k: x \mid P(q)} x^{-1} \sum_{y \mid P(q) x^{-1}} \mu(y) y^{-1}, \\
& =\sum_{x \leqq k ; x \leqq P(h)} x^{-1} \sum_{y \leqq P(h) x-1} \mu(y) y^{-1} \sum_{q \leqq h \cdot x y \mid P(q)} 1 .
\end{aligned}
$$


The number of $q \leqq h$ such that $x y \mid P(q)$ equals $h z(x y)(x y)^{-1}+O(z(x y))$. Therefore

$$
\begin{gathered}
T_{k, h}=h \sum_{x \leqq k ; x \leqq P(h)} \sum_{y \leqq P(h) x^{-1}} z(x y)(x y)^{-2} \mu(y)+O\left(\sum_{x \leqq k} \sum_{y \leqq P(h)} z(x y)(x y)^{-1}\right) \\
=h U_{k, h}+O\left(V_{k, h}\right),
\end{gathered}
$$

say. Putting $x y=w$ and using (4.1) with $\delta=\varepsilon / 2$ and Lemma 5 with $s=1+\varepsilon / 2$, $\varepsilon>0$, we find

$$
\begin{aligned}
U_{k, h} & =\sum_{w \leqq k ; w \leqq P(h)} z(w) w^{-2} \sum_{y \mid w} \mu(y)+O\left(\sum_{w>k} z(w) w^{-2} D(w)\right) \\
& =1+O\left(k^{\varepsilon-1}\left(\sum_{w=1}^{\infty} z(w) w^{-1-\varepsilon / 2} D(w) w^{-\varepsilon / 2}\right)\right)=1+O\left(k^{\varepsilon-1}\right) .
\end{aligned}
$$

Similarly,

$$
V_{k, h} \leqq \sum_{w \leqq P(h) k} z(w) w^{-1} D(w) \leqq P(h)^{8} k^{\varepsilon} \sum_{w=1}^{\infty}\left(z(w) w^{-1-\varepsilon / 2} D(w) w^{-\varepsilon / 2}\right) \ll P(h)^{8} k^{\varepsilon} .
$$

Combining our formulae and observing that $\varepsilon>0$ was arbitrary we obtain $T_{k, h}=h+O\left(h k^{\delta-1}+h^{\delta} k^{\delta}\right)$, thereby proving the proposition.

We use the remainder of this section to prove four related lemmas.

LemMa 6. Let $P(x)$ be a polynomial of degree $f>1$, and let $\varepsilon>0$. Then

$$
W_{h} \equiv \sum_{q=1}^{h} P(q)^{-1} \sum_{d \mid P(q) ; d<q^{f-.}} d \sum_{r \leqq q ; d \mid P(r)} 1 \ll h .
$$

Proof. Choose $\delta>0$ so small that $2 \delta f \leqq \varepsilon\left(1-f^{-1}\right)$.

There is an integer $g \geqq 1$ and a polynomial $Q(x)$ with no multiple factors such that $P(x) \mid Q(x)^{g}$. We may choose $g \leqq f$. Now $d \mid P(r)$ implies ${ }^{g}(d) \mid Q(r)$, hence the number of $r \leqq q$ with $d \mid P(r)$ is not larger than $\left(q\left({ }^{g}(d)\right)^{-1}+1\right) z_{Q}\left({ }^{g}(d)\right)$ and therefore by the corollary to Lemma 4 not larger than

$$
\ll\left(q\left({ }^{g}(d)\right)^{-1}+1\right) d^{\delta} \leqq\left(q d^{-1 / g}+1\right) d^{\delta} \leqq\left(q d^{-1 / \delta}+1\right) d^{\delta} .
$$

Using $D(P(q)) \ll q^{f \delta}$ we obtain

$$
\begin{aligned}
W_{h} & \ll \sum_{q=1}^{h} q^{-f} \sum_{d \mid P(q) ; d<q^{f-\varepsilon}}\left(q d^{1-1 / f+\delta}+d^{1+\delta}\right) \\
& \ll \sum_{q=1}^{h} D(P(q))\left(q^{-f+1+(1-1 / f+\delta)(f-\varepsilon)}+q^{-f+(1+\delta)(f-\varepsilon)}\right) \\
& \ll \sum_{q=1}^{h} q^{2 \delta f-\varepsilon(1-1 / f)} \ll h .
\end{aligned}
$$

LeMMA 7. Let $P(x)$ be arbitrary and $\delta>0$. Then $\sum_{q=1}^{h} \sum_{d \mid P(q)} d^{-\delta} \ll h$.

Froof. The part of the sum where $d \geqq q$ is not larger than 


$$
\sum_{q=1}^{h} q^{-\delta} D(P(q)) \ll h
$$

The part of the sum where $d<q$ is estimated by

$$
\sum_{d=1}^{h} d^{-\delta} \sum_{d<q \leqq h ; d \mid P(q)} 1 \leqq \sum_{d=1}^{h} d^{-\delta} h d^{-1} z(d) \leqq h \sum_{d=1}^{\infty} z(d) d^{-1-\delta} \ll h .
$$

LEMMA 8. Write $D_{k}(x)$ for the number of positive divisors of $x$ which are not larger than $k$, and let $\delta>0$. Then

$$
\sum_{q=1}^{h} D_{k}(P(q)) \ll h k^{\delta}
$$

Proof. We break the sum into two parts, $\sum_{q=1}^{\min (k, h)}+\Sigma_{k<q \leqq h}$, where the second part may be empty. For $q$ contributing to the first part of the sum, $D_{k}(P(q)) \leqq D(P(q)) \ll k^{\delta}$, and we obtain the desired estimate. The second part equals

$$
\begin{aligned}
\sum_{k<q \leqq h} D_{k}(P(q)) & =\sum_{d \leqq k} \sum_{k<q \leqq h ; d \mid P(q)} 1 \leqq \sum_{d \leqq k} h d^{-1} z(d) \\
& \leqq h k^{\delta} \sum_{d=1}^{\infty} z(d) d^{-1-\delta} \ll h k^{\delta} .
\end{aligned}
$$

LEMMA 9. Write $D(x, y)$ for the number of common positive divisors of integers $x, y \neq 0,0$. Let $P_{1}(x), P_{2}(x)$ be polynomials with integral coefficients such that $P_{i}(x) \neq 0$ for $x>0$. Then

$$
X_{h_{1}, h_{2}} \equiv \sum_{q_{1}=1}^{h_{1}} \sum_{q_{2}=1}^{h_{2}} D\left(P_{1}\left(q_{1}\right), P_{2}\left(q_{2}\right)\right) \ll h_{1} h_{2} .
$$

This estimate holds simultaneously in $h_{1}, h_{2}$.

Proof. It is sufficient to prove (5.1) with $P_{1}(x), P_{2}(x)$ both replaced by the product $P_{1}(x) P_{2}(x)$. We may therefore assume $P_{1}(x)=P_{2}(x)=P(x)$, say. There is an integer $g>0$ and a polynomial $Q(x)$ without multiple factors such that $P(x) \mid Q(x)^{g}$.

Let $\sigma$ be the set of positive divisors of $P(x)$ where $1 \leqq x \leqq \min \left(h_{1}, h_{2}\right)$. The number of elements of $\sigma$ is $\ll\left(\min \left(h_{1}, h_{2}\right)\right)^{1+\delta}$ for every $\delta>0$.

$$
\begin{aligned}
X_{h_{1}, h_{2}} & \leqq \sum_{d \in \sigma}\left(\sum_{q_{1} \leqq h_{1} ; d \mid P\left(q_{1}\right)} 1\right)\left(\sum_{q_{2} \leqq h_{2} ; d \mid P\left(q_{2}\right)} 1\right) \\
& \leqq \sum_{d=\sigma}\left(\sum_{q_{1} \leqq h_{1} ; g^{(d) \mid Q\left(q_{1}\right)}} 1\right)\left(\sum_{q_{2} \leqq h_{2} ; g(d) \mid Q(q) 2} 1\right) \\
& \leqq \sum_{d \in \sigma}\left(h_{1}\left({ }^{g}(d)\right)^{-1}+1\right)\left(h_{2}\left({ }^{g}(d)\right)^{-1}+1\right) z_{Q}^{2}\left({ }^{g}(d)\right) \\
& \ll \sum_{d \in \sigma}\left(h_{1}\left(\left(^{g}(d)\right)^{-1}+1\right)\left(h_{2}\left({ }^{g}(d)\right)^{-1}+1\right)\left({ }^{g}(d)\right)^{2 \delta} .\right.
\end{aligned}
$$


Using the distributive law we can break this sum into four parts, and Lemma 5 implies that each part is $\ll h_{1} h_{2}$.

6. Estimates for $A(k, q, r)$. In what follows, $d^{*}=d^{*}(q, r)$ will mean g.c.d. $(P(q), P(r))$. Put $B(k, q, r)$ for the number of pairs of integers $p, s, p \in S(k, q)$, $s \in S(k, r), 0 \leqq p<P(q)$, such that

$$
|P(q)(s+\theta)-P(r)(p+\theta)|<\min \left(d^{*}, P(q) \psi(r)\right) .
$$

LEMMA 10 . For $r \leqq q, A(k, q, r) \leqq \psi(q) P(q)^{-1} B(k, q, r)$.

Proof. All the expressions $P(q) s-P(r) p$ are multiples of $d^{*}$. Write $C(l, k, q, r)$ for the number of pairs $p, s, p \in S(k, q), s \in S(k, r), 0 \leqq p<P(q)$ such that $P(q) s-P(r) p=l d^{*}$. The congruence $P(r) p \equiv l d^{*}(\bmod P(q))$ has $d^{*}$ solutions in $p$, and therefore

$$
C(l, k, q, r) \leqq d^{*}
$$

By definition,

$$
\Gamma(k, q, r)=\sum_{p \in S(k, q)} \sum_{s \in S(k, r)} \int_{0}^{1} \beta(q, P(q) \alpha-p) \beta(r, P(r) \alpha-s) d \alpha .
$$

We now make the substitution $P(q) \alpha^{\prime}=P(q) \alpha-p-\theta$. Then $P(r) \alpha-s=P(r) \alpha^{\prime}+\theta-(P(q)(s+\theta)-P(r)(p+\theta)) P(q)^{-1}$ and

$\Gamma(k, q, r)$

$$
\begin{aligned}
& =\sum_{p \in S(k, q)} \sum_{s \in S(k, r)} \int_{-(p+\theta) P(q)^{-1}}^{1-(p+\theta) P(q)^{-1}} \quad \beta\left(q, P(q) \alpha^{\prime}+\theta\right) \beta\left(r, P(r) \alpha^{\prime}+\theta\right. \\
& =\sum_{l} C(l, k, q, r) \\
& \left.-(P(q)(s+\theta)-P(r)(p+\theta)) P(q)^{-1}\right) d \alpha^{\prime} \\
& \left.\cdot \int_{-\infty}^{\infty} \beta(q, P(q) \alpha+\theta) \beta\left(r, P(r) \alpha+\theta-l d^{*}+(P(q)-P(r)) \theta\right) P(q)^{-1}\right) d \alpha \\
& =\sum_{l} C(l, k, q, r) D\left(q, r, l d^{*}+(P(q)-P(r)) \theta\right),
\end{aligned}
$$

where $D(q, r, t)=\int_{-\infty}^{\infty} \beta(q, P(q) \alpha+\theta) \beta\left(r, P(r) \alpha+\theta-t P(q)^{-1}\right) d \alpha$.

For the following estimates we recall that $\beta(q, \xi+\theta)$ is the characteristic function of $0<\xi \leqq \psi(q)$. We note

$$
\int_{-\infty}^{\infty} D(q, r, t) d t=\psi(q) \psi(r)
$$

as well as $0 \leqq D(q, r, t) \leqq \psi(q) P(q)^{-1}$ and the fact that $D$ is zero outside the interval $(-P(q) \psi(r), P(r) \psi(q))$, hence in particular if $|t| \geqq P(q) \psi(r)$. Furthermore, $D(q, r, t)$ is decreasing for $t>0$, increasing for $t<0$. Hence 


$$
\begin{aligned}
\Gamma(k, q, r) \leqq & d^{*} \sum_{l ;\left|l d^{*}+(P(q)-P(r)) \theta\right| \geqq d^{*}} D\left(q, r, l d^{*}+(P(q)-P(r)) \theta\right) \\
& +\sum_{l ;\left|l d^{*}+(P(q)-P(r)) \theta\right|<d^{*}} C(l, k, q, r) D\left(q, r, l d^{*}+(P(q)-P(r)) \theta\right) \\
\leqq & d^{*} \int_{-\infty}^{\infty} D\left(q, r, \lambda d^{*}+(P(q)-P(r)) \theta\right) d \lambda+\psi(q) P(q)^{-1} B(k, q, r) \\
= & \psi(q) \psi(r)+\psi(q) P(q)^{-1} B(k, q, r),
\end{aligned}
$$

and the lemma follows.

Put $E_{\delta}(k, q, r)$ for the number of $p \in S(k, q), s \in S(k, r), 0 \leqq p<P(q)$ with $|P(q)(s+\theta)-P(r)(p+\theta)|<P(q) q^{-1} d^{* \delta}$.

LEMMA 11. Let $P(q)$ be a polynomial of degree $f>0$, and let $\varepsilon=1$ if $f=1$, $\varepsilon>0$ if $f>1$. Let $\delta>0$. Then

$$
\begin{aligned}
\sum_{q=1}^{h} \sum_{r=1}^{q} \psi(q) P(q)^{-1} B(k, q, r) \ll \Psi(h)^{1+\delta} & \\
& \quad+\sum_{q=1}^{h} \sum_{r \leqq q \text { with } d^{*} \geqq q^{f-r}} \psi(q) P(q)^{-1} E_{\delta}(k, q, r) .
\end{aligned}
$$

Proof. Choose $\delta_{1}>0, \delta_{2}>0$ such that $\delta_{1}+1 / \delta_{2}<\delta$. We shall use the easily proved estimate

$$
B(k, q, r) \leqq 2 d^{*} .
$$

We consider four parts $\Sigma_{1}, \Sigma_{2}, \Sigma_{3}, \Sigma_{4}$ of the sum we want to estimate.

$\Sigma_{1}$ : $d^{*}<q^{f-\varepsilon}$. We may assume $f>1$, since $d^{*}<q^{f-\varepsilon}$ is otherwise impossible.

$$
\begin{aligned}
\Sigma_{1} & \leqq 2 \sum_{q=1}^{h} \sum_{r \leqq q \text { with }} \sum_{d^{*}<q^{f-}} \psi(q) P(q)^{-1} d^{*} \\
& \leqq 2 \sum_{q=1}^{h} \psi(q) P(q)^{-1} \sum_{d \mid P(q) ; d<q^{f-}} d \sum_{r \leqq q ; d \mid P(r)} 1 .
\end{aligned}
$$

Using Lemma 6 and partial summation we obtain $\Sigma_{1} \ll \Psi(h)$.

$$
\Sigma_{2}: d^{*}<(q / r)^{1 / \delta_{1}}
$$

$$
\begin{aligned}
\sum_{r<q d^{*}-\delta_{1}} B(k, q, r) & \ll \sum_{d \mid P(q)} \sum_{r<q d^{-\delta_{1} ; d \mid P(r)}} d \leqq \sum_{d \mid P(q)} d \sum_{x \leqq P\left(q d^{\left.-\delta_{1}\right) ; d \mid x}\right.} 1 \\
& \ll \sum_{d \mid P(q)} d P(q) d^{-1-f \delta_{1}} . \\
\sum_{2} & \ll \sum_{q=1}^{h} \psi(q) \sum_{d \mid P(q)} d^{-f \delta_{1}} \ll \Psi(h)
\end{aligned}
$$

by Lemma 7 and partial summation. 
$\Sigma_{3}: d^{*}<\Psi(q)^{\delta_{2}}$.

$$
\begin{aligned}
\sum_{r<q \text { with } d^{*}<\Psi(q)} \delta_{2} B(k, q, r) & \ll \sum_{d \mid P(q) ; d<\Psi(q) \delta^{\delta_{2}}} \sum_{r \leqq q ; d \mid P(r)} d \\
& \leqq \sum_{d \mid P(q) ; d<\Psi(q)^{\delta_{2}}} d \sum_{x \leqq P(q) ; d \mid x} 1 \\
& \leqq \sum_{d \mid P(q) ; d<\Psi(q)^{\delta_{2}}} d P(q) d^{-1}=P(q) \underset{d \mid P(q) ; d<\Psi(q)^{\delta_{2}}}{ } 1 .
\end{aligned}
$$

Putting $l=\Psi(h)^{\delta_{2}}$ we obtain

$$
\Sigma_{3} \ll \sum_{q=1}^{h} \psi(q) D_{l}(P(q)) .
$$

Lemma 8 together with partial summation gives $\Sigma_{3} \ll \Psi(h)^{1+\delta}$.

$\Sigma_{4}: d^{*} \geqq q^{f-\varepsilon}, d^{*} \geqq(q / r)^{1 / \delta_{1}}, d^{*} \geqq \Psi(q)^{\delta_{2}}$. Under these conditions, $P(q) \psi(r) \leqq P(q) \psi\left(q d^{*-\delta_{1}}(3)=P(q) q^{-1} d^{* \delta_{1}} q d^{*-\delta_{1}} \psi\left(q d^{*-\delta_{1}}\right) \leqq P(q) q^{-1} d^{* \delta_{1}}\right.$ $\Psi(q) \leqq P(q) q^{-1} d^{* \delta_{1}+1 / \delta_{2}} \leqq P(q) q^{-1} d^{* \delta}$; therefore $B(k, q, r) \leqq E_{\delta}(k, q, r)$. Obviously, $\Sigma_{4}$ is bounded by the right-hand sum of (6.2).

7. Proof of Proposition 2 for nonlinear polynomials. In the case of polynomials of degree $f>1$ we may use Lemma 6 , which ceases to be true if $f=1$. On the other hand, much of the preceding discussion could be simplified for $f=1$.

We assume now $f>1$.

We define $(x, y ; k)$ by

$$
(x, y ; k)=\left\{\begin{array}{l}
\text { g.c.d. }(x, y) \text { if this divisor is } \geqq x k^{-1}, \\
0 \text { otherwise. }
\end{array}\right.
$$

Lemma 12. Assume that $\delta>0$ is so small that $f-1 / 2<(f-1 / 4)(1-\delta)$. Further assume $q>q_{0}(P, \delta), \quad r \leqq q, d^{*}=$ g.c.d. $(P(q), P(r)) \geqq q^{f-1 / 4}$. Then

$$
E_{\delta}(k, q, r) \leqq(P(q), P(r) ; k) .
$$

Proof. Put $c=\left|a_{0}\right|+\cdots+\left|a_{f}\right|$, where the $a_{i}$ 's are the coefficients of $P(q)$. Choose $q_{0}$ so large that the two inequalities

$$
q_{0}^{1 / 4}>2 c, \quad 2 c q_{0}^{f-1 / 2}<q_{0}^{(f-1 / 4)(1-\delta)}
$$

hold, and let $q \geqq q_{0}$.

The numbers $a=a(q), b=b(q)$ satisfy

$$
\theta=b / a+R,|R|<a^{-1} q^{-1 / 2}, a \leqq q^{1 / 2}
$$

and

$$
2 c a q^{f-1} \leqq 2 c q^{f-1 / 2}<q^{(f-1 / 4)(1-\delta)} \leqq d^{* 1-\delta} .
$$

(3) For $0 \leqq \alpha<1$ define $\psi(n-a)=\psi(n)$. 
$E_{\delta}(k, q, r)$ is bounded by the number of pairs $p, s, 0 \leqq p<P(q), p \in S(k, q)$ satisfying

$$
|P(q) s-P(r) p+(P(q)-P(r))(b / a+R)|<P(q) q^{-1} d^{* \delta} .
$$

This equation together with (7.2) yields

$$
\begin{aligned}
|P(q)(s+b / a)-P(r)(p+b / a)| & <P(q) q^{-1} d^{* \delta}+|P(q)-P(r)||R| \\
& \leqq c q^{f-1} d^{* \delta}+c q^{f} a^{-1} q^{-1 / 2} \\
& <d^{*} / 2 a+q^{f-1 / 4} / 2 a \leqq d^{*} / a .
\end{aligned}
$$

The left-hand side of (7.3) is an integral multiple of $d^{*} / a$, hence it must be zero.

$$
P(q)(a s+b)=P(r)(a p+b) .
$$

It remains to estimate the number of solutions of (7.4) in pairs $p, s, 0 \leqq p<P(q)$, $p \in S(k, q)$. Putting $P(q)=d^{*} P(q)^{*}$ we find that (7.4) implies

$$
a p+b \equiv 0\left(\bmod P(q)^{*}\right) .
$$

Since $a$ and $b$ are relatively prime, this congruence has at most one solution in $p$ modulo $P(q)^{*}$, hence at most $d^{*}$ solutions in $0 \leqq p<P(q)$. On the other hand, the congruence gives g.c.d. $(a p+b, P(q)) \geqq P(q)^{*}=P(q) d^{*-1}$, while $p \in S(k, q)$ implies g.c.d. $(a p+b, P(q)) \leqq k$. Thus $E_{\delta}(k, q, r)$ is zero unless $d^{*} \geqq P(q) k^{-1}$.

Lemma 12 is proved.

Proof of Proposition $2(f>1)$. We may assume that $\delta>0$ is so small that $f-1 / 2<(f-1 / 4)(1-\delta)$. Combining Lemma 10, Lemma 11 with $\varepsilon=1 / 4$ and Lemma 12 , we obtain

$$
\sum_{q=1}^{h} \sum_{r=1}^{h} A(k, q, r) \ll \Psi(h)^{1+\delta}+\sum_{q=1}^{h} \sum_{r=1}^{q} \psi(q) P(q)^{-1}(P(q), P(r) ; k) .
$$

Using Lemma 8 and partial summation we find

$$
\begin{aligned}
\sum_{q=1}^{h} \sum_{r=1}^{q} P(q)^{-1}(P(q), P(r) ; k) & \leqq \sum_{q=1}^{h} P(q)^{-1} \sum_{d \mid P(q) ; d \geqq P(q) k^{-1}} d \sum_{r \leqq q ; d \mid P(r)} 1 \\
& \leqq \sum_{q=1}^{h} P(q)^{-1} \sum_{d \mid P(q) ; d \geqq P(q) k^{-1}} d P(q) d^{-1} \\
& =\sum_{q=1}^{h} \sum_{d \mid P(q) ; d \leqq k} 1 \\
& =\sum_{q=1}^{h} D_{k}(P(q)) \ll h k^{\delta}
\end{aligned}
$$

and

$$
\sum_{q=1}^{h} \sum_{r=1}^{q} \psi(q) P(q)^{-1}(P(q), P(r) ; k) \ll \Psi(h) k^{\delta} .
$$


8. Proof of Proposition 2 for linear polynomials.

LEMma 13. Let $P(x)$ be a linear polynomial, let $0<\delta<1 / 4, q \geqq 1$ and $d^{*} \mid P(q)$. Then

$$
\sum_{r \leqq q ;(P(q), P(r))=d^{*}} E_{\delta}(k, q, r) \ll q d^{*-1 / 4}+d^{*}+q^{1 / 2}+\left\{\begin{array}{l}
q \text { if } d^{*} \geqq P(q) k^{-1}, \\
0 \text { otherwise. }
\end{array}\right.
$$

REMARK. The constant involved in the symbol $\ll$ depends on $P(x)$ only.

Proof. Put $c=\left|a_{0}\right|+\left|a_{1}\right|$ where $P(x)=a_{0} x+a_{1}$. The relation $|P(q) s-P(r) p+(P(q)-P(r)) \theta|<P(q) q^{-1} d^{* \delta} \leqq c d^{* 1 / 4}$ in the definition of $E_{\delta}(k, q, r)$ implies $\left\|(P(q)-P(r)) d^{*-1} \theta\right\|<c d^{*^{-3 / 4}}$. Given an $r$ such that the last inequality holds, there are at most $2 c$ integers $l$ with

$$
\left|(P(q)-P(r)) d^{*-1} \theta-l\right|<c d^{*-3 / 4} .
$$

Given $r$ and $l, P(q) s-P(r) p=l d^{*}$ has at most $d^{*}$ solutions in $p, 0 \leqq p<P(q)$. Putting

$$
F(q, r)=\left\{\begin{array}{l}
1 \text { if }\left\|(P(q)-P(r)) d^{*-1} \theta\right\|<c d^{*-3 / 4}, \\
0 \text { otherwise, }
\end{array}\right.
$$

we thus find

$$
E_{\delta}(k, q, r) \ll d^{*} F(q, r) .
$$

Assume now that $r$ runs through those values between 1 and $q$ where $d^{*} \mid P(r)$. Then $P(q)-P(r)$ runs through some or all of the numbers $0, d^{*}, 2 d^{*}, \cdots$, $\left[a_{0} q / d^{*}\right] d^{*}=q^{*} d^{*}$. Thus if we put $G\left(q, d^{*}\right)$ for the number of integers $x$ in $0 \leqq x \leqq q^{*}$ satisfying

$$
\|x \theta\|<c d^{*-3 / 4}
$$

then

$$
\sum_{r \leqq q ; d^{*} \mid P(r)} F(q, r) \leqq G\left(q, d^{*}\right) .
$$

We now distinguish three cases: $A, B$ and $C$.

A. $2 c a(q) \geqq d^{* 1 / 4}$. How often does (8.3) hold when $x$ runs through an interval $m<x \leqq m+a(q)$ ? Putting $\theta=b / a+R$ and $x=m+y$, the inequality becomes $\|m \theta+y b / a+y R\|<c d^{*-3 / 4}$ and this implies $\|m \theta+y b / a\|<c d^{*-3 / 4}$ $+a|R|<c d^{*-3 / 4}+q^{-1 / 2}$. The number of solutions of (8.3) for $x$ in an interval of length $a$ is therefore $\ll\left(d^{*-3 / 4}+q^{-1 / 2}\right) a+1 \ll d^{*-3 / 4} a+1$. Hence and

$$
G\left(q, d^{*}\right) \ll\left(d^{*-3 / 4} a+1\right)\left(q^{*} a^{-1}+1\right) \ll q^{*} d^{*-1 / 4}+q^{1 / 2} d^{*-3 / 4}+1,
$$

$$
d^{*}\left(G\left(q, d^{*}\right)\right) \ll q d^{*-1 / 4}+d^{*} .
$$

(8.1) now follows from (8.2), (8.4) and the last inequality. 
B. $2 c a(q)<d^{* 1 / 4}, 2 a q^{*}|R| \geqq 1$. Putting $\theta=b / a+R$ again, we rewrite (8.3) as $\|x b / a+x R\|<c d^{*-3 / 4}$. This implies that

$$
|m / a+x R|<c d^{*-3 / 4}
$$

for some integer $m$. For fixed $m$ the number of solutions in $x$ of (8.5) is at most $2 c d^{*-3 / 4}|R|^{-1}+1$. On the other hand, $x \leqq q^{*}$, whence $|m| \leqq c a d^{*-3 / 4}+a q^{*}|R|$. Thus

$$
\begin{aligned}
G\left(q, d^{*}\right) & \ll\left(d^{*-3 / 4}|R|^{-1}+1\right)\left(a d^{*-3 / 4}+a q^{*}|R|+1\right) \\
& \leqq a^{2} q^{*} d^{*-3 / 2}+a d^{*-3 / 4}+a q^{*} d^{*-3 / 4}+q^{*} q^{-1 / 2}+2 a q^{*} d^{*-3 / 4}+1 \\
& \ll a^{2} q^{*} d^{*-3 / 4}+q^{*} q^{-1 / 2}+1
\end{aligned}
$$

and

$$
d^{*} G\left(q, d^{*}\right) \ll q d^{*-1 / 4}+q^{1 / 2}+d^{*} .
$$

C. $2 c a(q)<d^{* 1 / 4}, 2 a q^{*}|R|<1$. $E_{\delta}(k, q, r)$ is bounded by the number of solutions in integers $p, s, 0 \leqq p<P(q), p \in S(k, q)$, of

$$
|P(q)(s+b / a)-P(r)(p+b / a)+(P(q)-P(r)) R|<c d^{* 1 / 4} .
$$

Now for $r \leqq q, d^{*} \mid P(r)$, one has $|P(q)-P(r)| \leqq q^{*} d^{*}$, and we obtain the inequality $|P(q)(s+b / a)-P(r)(p+b / a)| \leqq c d^{* 1 / 4}+q^{*} d^{*}|R|<d^{*} / 2 a+d^{*} / 2 a=d^{*} / a$.

Just as in the proof of Lemma 12 we may conclude that (7.4) holds, and we obtain (7.1). The number of $r \leqq q$ with $d^{*} \mid P(r)$ is $\ll q d^{*-1}$, and therefore

$$
\sum_{r \leqq q ; d^{*} \mid P(r)} E_{\delta}(k, q, r) \ll\left\{\begin{array}{l}
q \text { if } d^{*} \geqq P(q) k^{-1}, \\
0 \text { otherwise. }
\end{array}\right.
$$

Proof of Proposition $2(f=1)$. We may assume $0<\delta<1 / 4$. By applying Lemma 10 and Lemma 11 with $\varepsilon=1$ we obtain

$$
\sum_{q=1}^{h} \sum_{r=1}^{h} A(k, q, r) \ll \Psi(h)^{1+\delta}+\sum_{q=1}^{h} \sum_{r=1}^{q} \psi(q) P(q)^{-1} E_{\delta}(k, q, r) .
$$

By Lemma 13,

$$
\begin{aligned}
\sum_{q=1}^{h} & \sum_{r=1}^{h} P(q)^{-1} E_{\delta}(k, q, r) \\
& \ll \sum_{q=1}^{h} q^{-1} \sum_{d^{*} \mid P(q)}\left(q d^{*-1 / 4}+d^{*}+q^{1 / 2}\right)+\sum_{q=1}^{h} \sum_{d^{*} \mid P(q) ; d^{*} \geq P(q) k}{ }^{-1} 1 \\
& \ll \sum_{q=1}^{h} \sum_{d \mid P(q)} d^{-1 / 4}+\sum_{q=1}^{h} q^{-1 / 2} D(P(q))+\sum_{q=1}^{h} D_{k}(P(q)) \\
& \ll h+h+h k^{\delta} \ll h k^{\delta} .
\end{aligned}
$$


Here we used (4.1) and Lemmas 7 and 8 to estimate the last three sums. Proposition 2 now follows by partial summation.

9. The higher dimensional case. Most of the arguments used for the case $n=1$ carry over immediately to $n>1$, but some of them have to be modified.

We may assume that $I_{j q}$ is of the type $0<\left\{\xi_{j}-\theta_{j}\right\} \leqq \psi_{j}(q)$. For each of the integers $j=1, \cdots, n$ we can now define $a_{j}(q), b_{j}(b), S_{j}(k, q), \beta_{j}\left(q, \alpha_{j}\right)$, $\gamma_{j}\left(q, \alpha_{j}\right), \cdots, \Gamma_{j}(k, q, r)$. For given $q, r$ we write $d_{j}^{*}$ for the greatest common divisor of $P_{j}(g)$ and $P_{j}(r)$, and we may now define $B_{j}(k, q, r), \cdots, E_{j \delta}(k, q, r), F_{j}(q, r)$, $G_{j}\left(q, d_{j}^{*}\right)$. We put $\beta\left(q, \alpha_{1}, \cdots, \alpha_{n}\right)=\prod_{j} \beta_{j}\left(q, \alpha_{j}\right), \gamma\left(q, \alpha_{1}, \cdots, \alpha_{n}\right)=\prod_{j} \gamma_{j}\left(q, \alpha_{j}\right), \cdots$, $\Gamma(k, q, r)=\prod_{j} \Gamma_{j}(k, q, r)$, and we define $A(k, q, r)$ as in paragraph 2.

Proposition 1a. Let $\delta>0$. Then

$$
\sum_{q=1}^{h}\left(P_{1}(q) \cdots P_{n}(q)-\phi\left(k, P_{1}(q)\right) \cdots \phi\left(k, P_{n}(q)\right)\right)\left(P_{1}(q) \cdots P_{n}(q)\right)^{-1} \ll h k^{\delta-1}+h^{\delta} k^{\delta} \text {. }
$$

Proposition 2a. Let $\delta>0$. Then (3.2) holds.

The argument of paragraph 3 can be used to deduce the general theorem from these propositions.

Proposition 1a follows from Proposition 1 and

$$
\begin{aligned}
& P_{1}(q) \cdots P_{n}(q)-\phi\left(k, P_{1}(q)\right) \cdots \phi\left(k, P_{n}(q)\right) \\
& \quad=\sum_{j=1}^{n} P_{1} \cdots P_{j-1}\left(P_{j}-\phi\left(k, P_{j}\right)\right) \phi\left(k, P_{j+1}\right) \cdots \phi\left(k, P_{n}\right) .
\end{aligned}
$$

(6.1) now becomes

$$
\Gamma_{j}(k, q, r) \leqq \psi_{j}(q) \psi_{j}(r)+\psi_{j}(q) P_{j}(q)^{-1} B_{j}(k, q, r),
$$

and therefore for $r \leqq q$

$$
A(k, q, r) \leqq \sum_{m=1}^{n} \sum_{\Delta_{m}} H\left(k, q, r ; m, \Delta_{m}\right),
$$

where $\Delta_{m}$ runs through all divisions of the integers $1, \cdots, n$ into two classes $i_{1}, \cdots, i_{m}$ and $j_{1}, \cdots, j_{n-m}$, and where

$$
H\left(k, q, r ; m, \Delta_{m}\right)=\psi(q) \prod_{s=1}^{m}\left(P_{i_{s}}^{-1}(q) B_{i_{s}}(k, q, r)\right) \prod_{t=1}^{n-m} \psi_{j_{t}}(r) \text {. }
$$

For reasons of symmetry it will suffice to estimate $H\left(k, q, r ; m, \Delta_{m}^{0}\right)$, where $\Delta_{m}^{0}$ is the division with $i_{1}=1, \cdots, i_{m}=m$. We shall use

$$
B_{i}(k, q, r) \leqq 2 d_{i}^{*}
$$$$
(i=1, \cdots, n)
$$

LeMma 14. Let $m>1$. Then

$$
\sum_{q=1}^{h} \sum_{r=1}^{q} H\left(k, q, r ; m, \Delta_{m}^{0}\right) \ll \Psi(h) .
$$


Proof. We use the estimate

$$
H\left(k, q, r ; m, \Delta_{m}^{0}\right) \ll \psi(q) P_{1}(q)^{-1} P_{2}(q)^{-1} d_{1}^{*} d_{2}^{*} .
$$

By Schwartz' inequality,

$$
\begin{aligned}
Y_{h} & \equiv \sum_{q=1}^{h} \sum_{r=1}^{q} P_{1}(q)^{-1} d_{1}^{*} P_{2}(q)^{-1} d_{2}^{*} \\
& \leqq\left(\sum_{q=1}^{h} \sum_{r=1}^{q} P_{1}(q)^{-2} d_{1}^{* 2}\right)^{1 / 2}\left(\sum_{q=1}^{h} \sum_{r=1}^{q} P_{2}(q)^{-2} d_{2}^{* 2}\right)^{1 / 2} .
\end{aligned}
$$

Now

$$
\begin{aligned}
\sum_{q=1}^{h} \sum_{r=1}^{q} P(q)^{-2} d^{* 2} & \leqq \sum_{q=1}^{h} P(q)^{-2} \sum_{d \mid P(q)} d^{2} \sum_{r \leqq q ; d \mid P(r)} 1 \\
& \leqq \sum_{q=1}^{h} P(q)^{-2} \sum_{d \mid P(q)} d^{2} P(q) d^{-1}=\sum_{q=1}^{h} \sum_{d \mid P(q)} d^{-1} \ll h
\end{aligned}
$$

by Lemma 7 . Hence $Y_{h} \ll h$, and Lemma 14 follows by partial summation.

Everything can be completed as in the case $n=1$ once we have shown

LEMmA 11a. Let $\varepsilon=1$ if the degree $f_{1}$ of $P_{1}(x)$ equals $1, \varepsilon>0$ if $f_{1}>1$. Let $\delta>0$. Then

$$
\begin{aligned}
\sum_{q=1}^{h} \sum_{r=1}^{q} H\left(k, q, r ; 1, \Delta_{1}^{0}\right) & \\
& \ll \Psi(h)^{1+\delta}+\sum_{q=1}^{h} \sum_{r \leqq q \text { with } d_{1}^{*} \geqq q^{j_{1}--}} \psi(q) P_{1}(q)^{-1} E_{1 \delta}(k, q, r) .
\end{aligned}
$$

Proof. Choose $\delta_{1}>0, \delta_{2}>0$ such that $\delta_{1}+1 / \delta_{2}<\delta$. We write $\chi_{1}(q)=\psi_{2}(q) \cdots \psi_{n}(q)$ and put

$$
\begin{aligned}
& \Psi_{1}(h)=\sum_{q=1}^{h} \psi_{1}(q), \\
& X_{1}(h)=\sum_{q=1}^{h} \chi_{1}(q) .
\end{aligned}
$$

Since both $\psi_{1}(q)$ and $\chi_{1}(q)$ are decreasing, one has

$$
h \Psi(h) \geqq \Psi_{1}(h) \chi_{1}(h) .
$$

$H\left(k, q, r ; 1, \Delta_{1}^{0}\right)$ equals $\psi(q) P_{1}(q)^{-1} B_{1}(k, q, r) \chi_{1}(r)$.

We consider four parts of the sum we want to estimate. $\Sigma_{1}$ consists of terms with $d_{1}^{*}<q^{f_{1}-\varepsilon}, \Sigma_{2}$ of terms with $d_{1}^{*}<(q / r)^{1 / \delta_{1}}, \Sigma_{3}$ of terms where $d_{1}^{*}<\Psi_{1}(q)^{\delta_{2}}$, and $\Sigma_{4}$ consists of the remaining terms, that is, terms where $d_{1}^{*} \geqq q^{f_{1}-\varepsilon}$, 
$d_{1}^{*} \geqq(q / r)^{1 / \delta_{1}}, d_{1}^{*} \geqq \Psi_{1}(q)^{\delta_{2}}$. For the parts $\Sigma_{1}, \Sigma_{2}, \Sigma_{4}$ we estimate $H(\cdots) \leqq \psi(q) P_{1}(q)^{-1} B_{1}(k, q, r)$ and proceed as in paragraph 6 . The difficulty lies in estimating $\Sigma_{3}$.

Let $d \mid P_{1}(q)$ and denote the numbers $r$ having $r \leqq q$ and $d \mid P_{1}(r)$ by $r_{1}<r_{2}<\cdots<r_{j}$. One has $j \leqq P_{1}(q) d^{-1}$ and $r_{j} \geqq c(j d)^{1 / f_{1}} \geqq c^{\prime} d j P_{1}(q)^{-1} q$ for large $q$. Hence

$$
\begin{aligned}
\sum_{r \leqq q \text { with } d_{1}^{*}<\Psi_{1}(q)} \delta_{2} B_{1}(k, q, r) \chi_{1}(r) & \ll \sum_{d \mid P_{1}(q) ; d<\Psi_{1}(q)} \delta_{2} d \sum_{r \leqq q ; d \mid P_{1}(r)} \chi_{1}(r) \\
& \ll \sum_{d \mid P_{1}(q) ; d<\Psi_{1}(q)} \delta_{2} d \chi_{1}(q) P_{1}(q) q^{-1} d^{-1} \\
& =\chi_{1}(q) P_{1}(q) q^{-1} D_{f}\left(P_{1}(q)\right)
\end{aligned}
$$

with $f=f(q)=\Psi_{1}(q)^{\delta_{2}}$. Hence

$$
\Sigma_{3} \ll \sum_{q=1}^{h} \psi(q) q^{-1} X_{1}(q) D_{f}\left(P_{1}(q)\right) .
$$

We estimate the last sum in three parts, which overlap somewhat.

$T_{1}: \Psi(q) \geqq q^{1 / 4 \delta_{2}}$. Unless this part is empty, there is a largest $q \leqq h$ in $T_{1}$ say $q_{1}$. By (4.1),

$$
\begin{aligned}
T_{1} & \leqq \sum_{q=1}^{q_{1}} \psi(q) D\left(P_{1}(q)\right) \ll \sum_{q=1}^{q_{1}} \psi(q) q^{\delta / 4 \delta_{2}} \leqq \Psi\left(q_{1}\right) q_{1}^{\delta / 4 \delta_{2}} \\
& \leqq \Psi\left(q_{1}\right)^{1+\delta} \leqq \Psi(h)^{1+\delta} .
\end{aligned}
$$

$T_{2}: \chi_{1}(q) \leqq q^{1-1 / 4 \delta_{2}}$. Again using (4.1) we obtain

$$
T_{2} \ll \sum_{q=1}^{h} \psi(q) q^{-1 / 4 \delta_{2}} q^{1 / 4 \delta_{2}}=\Psi(h) .
$$

$T_{3}: \Psi(q)<q^{1 / 4 \delta_{2}}, X_{1}(q)>q^{1-1 / 4 \delta_{2}}$. We denote the set of $q$ 's involved by $\sigma$, by $h_{1}$ the largest element of $\sigma$, and we write $g(q)=\psi(q) q^{-1} \chi_{1}(q)$. Observing (9.2) we find $\Psi_{1}(q) \leqq q^{1 / 2 \delta_{2}}$ and $f(q) \leqq q^{1 / 2}$ for $q \in \sigma$.

$$
T_{3}=\sum_{q \in \sigma} g(q) D_{f}\left(P_{1}(q)\right)=\sum_{d \leqq f\left(h_{1}\right)} \sum_{q \in \sigma: d \mid P_{1}(q) ; f(q) \geqq d} g(q) .
$$

Let $x_{1}, \ldots, x_{2(d)}$ be the solutions of $P_{1}(x) \equiv 0(\bmod d)$. Since $g(q)$ is decreasing, one has

$$
\sum_{q \in \sigma ; f(q) \geqq d ; q \equiv x_{i}(\bmod d)} g(q) \leqq g\left(q_{i}\right)+d^{-1} \sum_{f(q) \geqq d} g(q),
$$

where $q_{i}$ is the smallest $q \equiv x_{i}(\bmod d)$ such that $q \in \sigma$ and $f(q) \geqq d$. Since $f(q) \leqq q^{1 / 2}$, one finds $q_{i} \geqq d^{2}$. Therefore

$$
\sum_{q \varepsilon \sigma \cdot f(q) \leqq d ; d \mid P_{1}(q)} g(q) \leqq z(d) g\left(d^{2}\right)+z(d) d^{-1} \quad \sum_{f(q) \geqq d} g(q) .
$$


Observing $z(d) g\left(d^{2}\right) \leqq d g\left(d^{2}\right) \leqq \sum_{q=(d-1)^{2}+1}^{d^{2}} g(q)$ we obtain

$$
\begin{aligned}
T_{3} & \leqq \sum_{d \leqq f\left(h_{1}\right)}\left(\sum_{q=(d-1)^{2}+1}^{d^{2}} g(q)+z(d) d^{-1} \sum_{f(q) \geqq d} g(q)\right) \\
& \leqq \sum_{q=1}^{f^{2}\left(h_{1}\right)} \psi(q)+\sum_{q=1}^{h} g(q) \sum_{d \leqq f(q)} z(d) d^{-1} \\
& \ll \Psi(h)+\sum_{q=1}^{h} g(q) \Psi_{1}(q)^{\delta}
\end{aligned}
$$

by Lemma 5. (9.2) finally yields

$$
T_{3} \ll \Psi(h)+\sum_{q=1}^{h} \psi(q)\left(q^{-1} \chi_{1}(q)\right)^{1-\delta} \Psi(q)^{\delta} \ll \Psi(h)^{1+\delta} .
$$

Lemma 11a is proved.

10. Linear forms. We restrict ourselves to the case of one form only.

Proposition 3. Let $P_{1}\left(q_{1}\right), \cdots, P_{n}\left(q_{n}\right)$ be nonconstant polynomials, $n+1$, and let $I_{q_{1}, \ldots, q_{n}}$ be intervals of $C\left(q_{i}=1,2, \cdots ; i=1, \cdots, n\right)$. We assume that the length of $I_{q_{1}, \ldots, q_{n}}$ is $\psi_{1}\left(q_{1}\right) \psi_{2}\left(q_{2}\right) \cdots \psi_{n}\left(q_{n}\right)$, where $\psi_{i}(x)$ are decreasing functions $(i=1, \cdots, n)$, and we put

$$
\Psi_{i}(h)=\sum_{q_{i}=1}^{h} \psi_{i}\left(q_{i}\right)
$$

We write $M\left(h_{1}, \cdots, h_{n} ; \alpha_{1}, \cdots, \alpha_{n}\right)$ for the number of solutions of $\left\{\alpha_{1} P_{1}\left(q_{1}\right)+\cdots+\alpha_{n} P_{n}\left(q_{n}\right)\right\} \in I_{q_{1}, \ldots, q_{n}}$, where $1 \leqq q_{i} \leqq h_{i}(i=1, \cdots, n)$. Let $\varepsilon>0$. Then for almost all $\alpha_{1}, \cdots, \alpha_{n}$,

$$
M\left(h_{1}, \cdots, h_{n} ; \alpha_{1}, \cdots, \alpha_{n}\right)=\Psi_{1}\left(h_{1}\right) \cdots \Psi_{n}\left(h_{n}\right)+O\left(\Psi_{1}\left(h_{1}\right) \cdots \Psi_{n}\left(h_{n}\right)\right)^{1 / 2+\varepsilon} .
$$

This estimate holds simultaneously for $h_{1}, \cdots, h_{n}$.

Proof. We restrict ourselves to a few hints. The reader might compare paragraph 6 of [12]. We assume $n>1$.

We put $\beta\left(q_{1}, \cdots, q_{n}, \xi\right)$ equal to 1 if $\{\xi\} \in I_{q_{1}, \ldots, q_{n}}$ and $\xi \in U, \beta(\cdots)=0$ otherwise. $\Gamma\left(q_{1}, \cdots, q_{n} ; r_{1}, \cdots, r_{n}\right)$ stands for the integral

$\int_{0}^{1} \cdots \int_{0}^{1}\left(\sum_{p}\left(\beta q_{1}, \cdots, q_{n}, \Sigma \alpha_{i} P_{i}\left(q_{i}\right)-p\right)\right)\left(\sum_{s} \beta\left(r_{1}, \cdots, r_{n}, \sum \alpha_{i} P_{i}\left(r_{i}\right)-s\right)\right) d \alpha_{1} \cdots d \alpha_{n}$, and $A\left(q_{1}, \cdots, q_{n} ; r_{1}, \cdots, r_{n}\right)$ for

$$
\Gamma\left(q_{1}, \cdots, r_{n}\right)-\psi_{1}\left(q_{1}\right) \cdots \psi_{n}\left(q_{n}\right) \psi_{1}\left(r_{1}\right) \cdots \psi_{n}\left(r_{n}\right) .
$$

PROPOSITION 2b. $\sum_{q_{1}=1}^{h_{1}} \cdots \sum_{r_{n}=1}^{h} A\left(q_{1}, \cdots, r_{n}\right) \ll \Psi_{1}\left(h_{1}\right) \cdots \Psi_{n}\left(h_{n}\right)$. 
To deduce Proposition 3 from Proposition $2 b$ we put

$$
M\left(h_{1}, \cdots, h_{n} ; k_{1}, \cdots, k_{n} ; \alpha_{1}, \cdots, \alpha_{n}\right)
$$

for the number of $q_{1}, \cdots, q_{n}, h_{i}<q_{i} \leqq k_{i}(i=1, \cdots, n)$ such that $\left\{\sum \alpha_{i} P_{i}\left(q_{i}\right)\right\} \in I_{q_{1}, \ldots, q_{n}}$ and we put $\Psi_{i}(h, k)=\Sigma_{h<q \leqq k} \psi_{i}(q)$. We choose integers $m_{u}^{i}=m_{u}^{i}\left(r_{1}, \cdots, r_{n}\right)$ such that $\left[2^{r_{1}+\ldots+r_{n}-r_{i}} \Psi_{i}\left(m_{u}^{i}\right)\right]=u$ The following two lemmas are now used.

Lemma $2 b$. Let $\delta>0$. Then one has for $T=T_{r_{1}+\ldots+r_{n}}$

$$
\begin{gathered}
\sum_{\left(u_{1} v_{1}\right] \in J} \ldots \sum_{\left(u_{n} v_{n}\right] \in J} \int_{0}^{1}\left(M \left(m_{u_{1}}^{1}, \cdots,\right.\right. \\
\left.-m_{u_{n}}^{n} ; m_{v_{n}}^{1}, \cdots, m_{v_{n}}^{n} ; \alpha_{1}, \cdots, \alpha_{n}\right) \\
\left.-\Psi_{1}\left(m_{u_{1}}^{1}, m_{v_{1}}^{1}\right) \cdots \Psi_{n}\left(m_{u_{n}}^{n}, m_{v_{n}}^{n}\right)\right)^{2} d \alpha_{1} \cdots d \alpha_{n} \\
\ll 2^{\left(r_{1}+\ldots+r_{n}\right)(1+\delta)} .
\end{gathered}
$$

Lemma 3b. Let $\delta>0$. There are subsets $\sigma_{r_{1}, \ldots, r_{n}}\left(r_{i}=1,2, \cdots ; i=1, \cdots, n\right)$ of $U \times \cdots \times U$ with measures

$$
\mu_{r_{1}, \ldots, r_{n}} \ll r_{1}^{-2} \cdots r_{n}^{-2}
$$

such that

$$
M\left(m_{w_{1}}^{1}, \cdots, m_{w_{n}}^{n} ; \alpha_{1}, \cdots, \alpha_{n}\right)=\Psi_{1}\left(m_{w_{1}}^{1}\right) \cdots \Psi_{n}\left(m_{w_{n}}^{n}\right)+O\left(r_{1}^{2} \cdots r_{n}^{2} 2^{\left(r_{1}+\ldots+r_{n}\right)(1 / 2+\delta)}\right)
$$

for every $w_{1}, \cdots, w_{n}$ with $w_{i} \leqq 2^{r_{1}+\ldots+r}(i=1, \cdots, n)$ and $\left(\alpha_{1}, \cdots, \alpha_{n}\right)$ in $U \times \cdots \times U$ but not in $\sigma_{r_{1}, \ldots, r . .}$.

To prove Proposition $2 \mathrm{~b}$ we require

LEMMA 10b. A. If the matrix

has rank 2, then

$$
\left(\begin{array}{l}
P_{1}\left(q_{1}\right), \cdots, P_{n}\left(q_{n}\right) \\
P_{1}\left(r_{1}\right), \cdots, P_{n}\left(r_{n}\right)
\end{array}\right)
$$

$$
A\left(q_{1}, \cdots, r_{n}\right)=0 .
$$

B. If the matrix has rank 1 , then

$$
A\left(q_{1}, \cdots, r_{n}\right) \leqq \psi_{1}\left(q_{1}\right) \cdots \psi_{n}\left(q_{n}\right) P_{1}\left(q_{1}\right)^{-1} B_{1}\left(q_{1}, \cdots, r_{n}\right),
$$

where $B_{1}\left(q_{1}, \cdots, r_{n}\right)$ is the number of solutions of $\left|P_{1}\left(q_{1}\right)\left(s+\theta^{\prime}\right)-P_{1}\left(r_{1}\right)(p+\theta)\right|<d_{1}^{*}$ in integers $p, s, 0 \leqq p<P_{1}\left(q_{1}\right)$, where $\theta, \theta^{\prime}$ are the left endpoints of $I_{q_{1}, \ldots, q_{n}}, I_{r_{1}, \ldots, r_{n}}$, respectively, and where $d_{1}^{*}=$ g.c.d. $\left(P_{1}\left(q_{1}\right), P_{1}\left(r_{1}\right)\right)$.

We leave the proof of $\mathrm{A}$ to the reader. As for $\mathrm{B}$, we make the substitution $\alpha_{2}=\xi_{2}, \cdots, \alpha_{n}=\xi_{n}, \sum \alpha_{i} P_{i}\left(q_{i}\right)=\xi_{1} P_{1}\left(q_{1}\right)$, hence $\sum \alpha_{i} P_{i}\left(r_{i}\right)=\xi_{1} P_{1}\left(r_{1}\right)$. When $\xi_{2}, \cdots, \xi_{n}$ is fixed, $\xi_{1}$ ranges in an interval of length 1 , and $\Gamma$ equals 


$$
\int_{0}^{1}\left(\sum_{p} \beta\left(q_{1}, \cdots, q_{n}, \xi_{1} P_{1}\left(q_{1}\right)-p\right)\right)\left(\sum_{s} \beta\left(r_{1}, \cdots, r_{n}, \xi_{1} P_{1}\left(r_{1}\right)-s\right)\right) d \xi_{1} .
$$

This one-dimensional integral can be estimated by the method of paragraph 6 .

The proof of Proposition $2 \mathrm{~b}$ now proceeds as follows. We may restrict ourselves to terms $r_{1} \leqq q_{1}$. For fixed $q_{1}, \cdots, q_{n}$, let $\Delta=$ g.c.d. $\left(P_{1}\left(q_{1}\right), \cdots, P_{n}\left(q_{n}\right)\right)$ and $P_{i}\left(q_{i}\right)=P_{i}\left(q_{i}\right)^{*} \Delta(i=1, \cdots, n)$. In view of Lemma $10 \mathrm{~b}$ we may restrict ourselves to $r_{1} \leqq q_{1}$ where $P_{1}\left(r_{1}\right)$ is of the type $l P_{1}\left(q_{1}\right)^{*}$, whence $d_{1}^{*}=P_{1}\left(q_{1}\right)^{*}(\Delta, l)$. Since $B\left(q_{1}, \cdots, r_{n}\right) \leqq 2 d_{1}^{*}$, one has

$$
\begin{aligned}
& \sum_{q_{1}=1}^{h_{1}} \ldots \sum_{r_{n}=1}^{h_{n}} A\left(q_{1}, \cdots, r_{n}\right) \leqq 4 \sum_{q_{1}=1}^{h_{1}} \ldots \sum_{q_{n}=1}^{h_{n}} \psi_{1}(q)_{1} \cdots \psi_{n}\left(q_{n}\right) \sum_{l=1}^{\Delta} P_{1}\left(q_{1}\right)^{-1}\left(P_{1}\left(q_{1}\right)^{*}(\Delta, l)\right) \\
& \leqq 4 \sum_{q_{1}=1}^{h_{1}} \ldots \sum_{q_{n}=1}^{h_{n}} \psi_{1}\left(q_{1}\right) \cdots \psi_{n}\left(q_{n}\right) D(\Delta) \\
& \leqq 4\left(\sum_{q_{1}=1}^{h_{1}} \sum_{q_{2}=1}^{h_{2}} \psi_{1}\left(q_{1}\right) \psi_{2}\left(q_{2}\right) D\left(P_{1}\left(q_{1}\right), P_{2}\left(q_{2}\right)\right)\right) \\
& \Psi_{3}\left(h_{3}\right) \cdots \Psi_{n}\left(h_{n}\right) .
\end{aligned}
$$

Using Lemma 9 and partial summation both for the sum over $q_{1}$ and over $q_{2}$ we obtain

$$
\sum_{q_{1}=1}^{h_{1}} \sum_{q_{2}=1}^{h_{2}} \psi_{1}\left(q_{1}\right) \psi_{2}\left(q_{2}\right) D\left(P_{1}\left(q_{1}\right), P_{2}\left(q_{2}\right)\right) \ll \Psi_{1}\left(h_{1}\right) \Psi_{2}\left(h_{2}\right) .
$$

11. Theorem 2. To prove the lower bound in (1.5) we shall need

Proposition 4. Let $a(1)<a(2)<\cdots$ be a sequence of positive integers and put $M_{I}(h ; \alpha)$ for the number of $q \leqq h$ such that $\{\alpha a(q)\} \in I$. Then for $\varepsilon>0$ and almost all $\alpha$ the inequality

$$
\left|M_{I}(h ; \alpha)-h l(I)\right|<h^{1 / 2} \log ^{5 / 2+\varepsilon} h
$$

holds for all intervals $I$ and all $h>h_{1}$, where $h_{1}$ depends only on $\alpha$ and $\varepsilon$ (but not on I).

Proof. This proposition is a special case of Theorem 1 of [3] and of Theorem 1 of [6].

Proof of Theorem 2. We use the abbreviation

$$
\|\Sigma\|=\left\|\sum_{i=1}^{n} \alpha_{i} a_{i}\left(q_{i}\right)+\theta\right\| \text {. }
$$

Put $\delta=\varepsilon /(n+1)$. Using an idea of Littlewood [4, Appendix A], we consider the integral

$$
J\left(q_{1}, \cdots, q_{n}\right)=\int_{0}^{1} \cdots \int_{0}^{1}\left(\|\Sigma\||\log \|\Sigma\||^{1+\delta}\right)^{-1} d \alpha_{1} \cdots d \alpha_{n} .
$$


This integral has a finite value independent of $q_{1}, \cdots, q_{n}$. Hence the sum

$$
\sum_{q_{1}=1}^{\infty} \cdots \sum_{q_{n}=1}^{\infty}\left(q_{1} \log ^{1+\delta} q_{1} \cdots q_{n} \log ^{1+\delta} q_{n}\right)^{-1} J\left(q_{1}, \cdots, q_{n}\right)
$$

is convergent and

$$
\sum_{q_{1}=1}^{\infty} \cdots \sum_{q_{.}=1}^{\infty}\left(q_{1} \log ^{1+\delta} q_{1} \cdots q_{n} \log ^{1+\delta} q_{n}\|\Sigma\||\log \|\Sigma\||^{1+\delta}\right)^{-1}
$$

is convergent for almost all $\alpha_{1}, \cdots, \alpha_{n}$.

It is easy to see that the inequality

$$
\|\Sigma\| \leqq\left(q_{1} \cdots q_{n}\right)^{-2}
$$

has only a finite number of solutions in integers $q_{1}, \ldots, q_{n}$ for almost every $\alpha_{1}, \cdots, \alpha_{n}$.

We are going to show that the upper estimate for $\Sigma\left(h ; \alpha_{1}, \cdots, \alpha_{n}\right)$ in (1.5) is true for every $\alpha_{1}, \cdots, \alpha_{n}$ such that (11.1) is convergent and (11.2) has only finitely many solutions. There is a constant $c>0$ such that $\|\Sigma\| \geqq c^{-1}\left(q_{1} \cdots q_{n}\right)^{-2}$, whence

We obtain

$$
|\log \|\Sigma\|| \leqq 2 \log \left(q_{1} \cdots q_{n}\right)+\log c .
$$

$$
\begin{gathered}
\sum\left(h ; \alpha_{1}, \cdots, \alpha_{n}\right) \leqq\left(\max _{q_{i} \leqq h}\left(\log ^{1+\delta} q_{1} \cdots \log ^{1+\delta} q_{n}|\log \|\Sigma\||^{1+\delta}\right)\right) \\
\cdot \sum_{q_{1}=1}^{\infty} \cdots \sum_{q=1}^{\infty}\left(q_{1} \log ^{1+\delta} q_{1} \cdots q_{n} \log ^{1+\delta} q_{n}\|\Sigma\||\log \|\Sigma\||^{1+\delta}\right)^{-1} \\
\ll(\log h)^{(1+\delta) n}\left(\log h^{n}\right)^{1+\delta} \ll(\log h)^{n+1+\varepsilon} .
\end{gathered}
$$

We now turn to the proof of the lower bound in (1.5). We are going to apply Proposition 4 to the sequence $a(q)=a_{1}(q)$. For almost all reals $\alpha$ and $h \geqq h_{1}(\alpha)$ one has $\left|M_{I}(h ; \alpha)-h l(I)\right|<h^{3 / 4}$. Let $\alpha_{1}$ have this property. Denote the number of $q \leqq h$ such that $\left\|\alpha_{1} a_{1}(q)+\eta\right\| \leqq \gamma$ by $M_{\gamma, \eta}\left(h ; \alpha_{1}\right)$. Then

$$
\left|M_{\gamma, \eta}\left(h ; \alpha_{1}\right)-2 \gamma h\right|<h^{3 / 4} \quad\left(h \geqq h_{1}\left(\alpha_{1}\right), 0 \leqq \gamma \leqq 1 / 2, \eta \text { arbitrary }\right) .
$$

Let $k_{0}=k_{0}(h)$ be the largest integer with $2^{k_{0}+1} \leqq h^{1 / 4}$. Then $k_{0} \geqq 0$ for $h \geqq h_{2}\left(\alpha_{1}\right)=\max \left(h_{1}\left(\alpha_{1}\right), 2^{8}\right)$. The number $N_{k, \eta}\left(h ; \alpha_{1}\right)$ of $q \leqq h$ such that

$$
2^{-k-1}<\left\|\alpha_{1} a_{1}(q)+\eta\right\| \leqq 2^{-k}
$$

satisfies $N_{k, \eta}\left(h ; \alpha_{1}\right) \geqq 2^{-k} h-2 h^{3 / 4} \geqq 2^{-k-1} h$ for every $k$ in $0 \leqq k \leqq k_{0}$.

By considering the parts of the sum where (11.3) is satisfied for $k=0, \cdots, k_{0}$ we obtain

$$
\sum_{q=1}^{h}\left\|\alpha_{1} a_{1}(q)+\eta\right\|^{-1} \geqq \sum_{k=0}^{k_{0}} 2^{k} 2^{-k-1} h>\frac{1}{2} h k_{0}(h) \geqq c_{1}\left(\alpha_{1}\right) h \log h .
$$

Partial summation yields 


$$
\sum_{q=1}^{h}\left\|\alpha_{1} a_{1}(q)+\eta\right\|^{-1} q^{-1} \geqq c_{2}\left(\alpha_{1}\right) \log ^{2} h .
$$

This inequality holds for arbitrary $\eta$. By writing $\eta=\alpha_{2} a_{2}\left(q_{2}\right)+\cdots+\alpha_{n} a_{n}\left(q_{n}\right)+\theta$ and taking the sum over $q_{2}, \cdots, q_{n}$ one finds

$$
\Sigma\left(h ; \alpha_{1}, \cdots, \alpha_{n}\right) \geqq c_{3}\left(\alpha_{1}\right) \log ^{n+1} h .
$$

REMARK. Our method could be used to show the following: The left inequality of (1.5) is true for arbitrary $\alpha_{1}, \cdots, \alpha_{n-1}$ and $\alpha_{n} \in \sigma\left(\alpha_{1}, \cdots, \alpha_{n-1}\right)$, where $\sigma(\cdots)$ is a set containing almost all numbers. The other inequality of (1.5) holds for $n$-tuples such that $\alpha_{n} \in \tau$ where $\tau$ is independent of $\alpha_{1}, \cdots, \alpha_{n-1}$ and contains almost all numbers.

12. Theorem 3. We define a function $\pi\left(k_{1}, \cdots, k_{n}\right)$ as follows. $\pi(0, \cdots, 0)=0$, and if $k_{i_{1}}, \cdots, k_{i_{m}}$ are those $k_{i}$ 's which are different from zero, then $\pi\left(k_{1}, \cdots, k_{n}\right)=\left|k_{i_{1}} \cdots k_{i_{m}}\right|^{-1}$. In our applications $k_{1}, \cdots, k_{n}$ will always be integers. Write $\exp \xi$ for $e^{2 \mu i \xi}$.

GENERALIZED THEOREM OF ERDöS AND TURAN. There are absolute constants $c_{n}, n=1,2, \cdots$ with the following properties.

Let $n \geqq 1, h \geqq 1$, and let vectors $\left(\alpha_{1 q}, \cdots, \alpha_{n q}\right)$ be given $(q=1, \cdots, h)$. Put

$$
\omega\left(k_{1}, \cdots, k_{n}\right)=\left|\sum_{q=1}^{h} \exp \left(\sum_{i=1}^{n} \alpha_{i q} k_{i}\right)\right| .
$$

Let $I_{1}, \cdots, I_{n}$ be intervals of $C$ of lengths $l\left(I_{j}\right)=\psi_{j}$ and put $\psi=\prod \psi_{j}$. Write $N$ for the number of $q, 1 \leqq q \leqq h$, such that simultaneously $\left\{\alpha_{j q}\right\} \in I_{j}(j=1, \cdots, n)$. Let $m$ be a positive integer. Then

$$
|N-\psi l| \leqq c_{n}\left(h m^{-1}+\sum_{k_{1}, \ldots, k_{\ldots}:\left|k_{j}\right| \leqq m} \pi\left(k_{1}, \cdots, k_{n}\right) \omega\left(k_{1}, \cdots, k_{n}\right)\right) .
$$

This theorem is a generalization to $n$ dimensions of a result of Erdös and Turan [7, Theorem 3]. We shall not give a proof, since the argument in [7] can easily be extended to our situation.

Proof of Theorem 3. Put $\alpha_{j q}=\alpha_{j} q(j=1, \cdots, n ; q=1,2, \cdots)$.

$$
\omega_{h}\left(k_{1}, \cdots, k_{n}\right) \equiv\left|\sum_{q=1}^{h} \exp \left(\sum k_{i} \alpha_{i} q\right)\right| \ll\left\|k_{1} \alpha_{1}+\cdots+k_{n} \alpha_{n}\right\|^{-1} .
$$

Theorem 3 is an immediate consequence of the generalized Erdös-Turan Theorem with $m=h$ and the fact that

$$
\sum_{k_{1}, \ldots, k_{. .}:\left|k_{i}\right| \leqq m} \pi\left(k_{1}, \cdots, k_{n}\right)\left\|k_{1} \alpha_{1}+\cdots+k_{n} \alpha_{n}\right\|^{-1} \ll(\log m)^{n+1+\varepsilon}
$$

for almost every $\alpha_{1} \cdots, \alpha_{n}$. This fact follows from Theorem 2 . 


\section{REFERENCES}

1. H. Behnke, Zur Theorie der diophantischen Approximationen, Abh. Math. Sem. Univ. Hamburg 3 (1924), 261-318.

2. J. W. S. Cassels, Some metrical theorems in diophantine approximation. I, Proc. Cambridge Philos. Soc. 46 (1950), 209-218.

3. - Some metrical theorems in diophantine approximation. III, Proc. Cambridge Philos. Soc. 46 (1950), 219-225.

4. J. W. S. Cassels and H. P. F. Swinnerton-Dyer, On the product of three homogeneous linear forms and indefinite ternary quadratic forms, Philos. Trans. Roy. Soc. London A 248 (1956), 73-96.

5. P. Erdös, Some results on diophantine approximation, Acta Arith. 5 (1959), 359-369.

6. P. Erdös, and J. F. Koksma, On the uniform distribution modulo 1 of sequences $f(n, \theta)$, Indag. Math. 11 (1949), 299-302.

7. P. Erdös and P. Turan, On a problem in the theory of uniform distribution, Indag. Math. 10 (1948), 370-382 and 406-413.

8. A. Khintchine, Ein Satz über Kettenbrüche, mit arithmetischen Anwendungen, Math. Z. 18 (1923), 289-306.

9. ——, Einige Sätze über Kettenbrüche mit Anwendugen auf die Theorie der diophantischen Approximationen, Math. Ann. 92 (1924), 115-125.

10. W. J. Le Veque, On the frequency of small fractional parts in certain real sequences. III, J. Reine Angew. Math. 202 (1959), 215-220.

11. T. Nagell, Introduction to number theory, Wiley, New York, 1951.

12. W. Schmidt, A metrical theorem in diophantine approximation, Canad. J. Math. 12 (1960), 619-631.

13. P. Szüsz, Über die metrische Theorie der diophantischen Approximationen, Acta Math. Acad. Sci. Hungar. 9 (1958), 177-193.

\section{COlumbia University, NeW YORK, NeW YoRK \\ UNIVERSITY OF COLORADO, BOULDER, COLORADO}

